\title{
A chloroplast envelope-bound PHD transcription factor mediates chloroplast signals to the nucleus
}

Xuwu Sun', Peiqiang Feng ${ }^{1}$ Xiumei Xu', Hailong Guo', Jinfang Ma', Wei Chi', Rongchen Lin', Congming Lu' \& Lixin Zhang ${ }^{1}$

Chloroplast development, maintenance and function depend on the coordinated expression of chloroplast and nuclear genes. The retrograde chloroplast signals are essential in coordinating nuclear gene expression. Although the sources of signals in chloroplasts have been identified and the associated transcription factors in the nucleus extensively studied, the molecular mechanism that relays chloroplast signals to the nucleus remains a mystery. Here we show that PTM, a chloroplast envelope-bound plant homeodomain (PHD) transcription factor with transmembrane domains, functions in multiple retrograde signal pathways. The proteolytic cleavage of PTM occurs in response to retrograde signals and amino-terminal PTM accumulates in the nucleus, where it activates ABI4 transcription in a PHD-dependent manner associated with histone modifications. These results provide a molecular basis for the critical function of PTM in retrograde chloroplast signaling and shed new light on the mechanism whereby chloroplast signals are transmitted to the nucleus through the cytosol. 
C hloroplasts have essential roles in photosynthesis and associated metabolic pathways; therefore, they are of fundamental importance for life on the Earth. The chloroplast has its own genome, which encodes only $\sim 100$ genes, although more than 3,000 proteins function within the chloroplast ${ }^{1}$. Therefore, more than $95 \%$ of chloroplastic proteins are encoded by the nuclear genome, translated in the cytosol and post-translationally imported into the chloroplast $\mathrm{t}^{2-5}$. The chloroplast-encoded and imported nuclearencoded proteins are assembled to form functional photosynthetic and metabolic complexes ${ }^{6}$. Thus, tightly coordinated gene expression between the chloroplast and nuclear genomes is essential for chloroplast biogenesis and maintenance as well as for sustaining the reactions of photosynthesis. Chloroplast development and gene expression are regulated mainly by nuclear-encoded proteins, but retrograde signals from the chloroplast to the nucleus modulate the expression of nuclear genes encoding chloroplast-localized proteins to maintain the functional and metabolic states of the organelle $e^{7-12}$.

Evidence that signals originate from chloroplasts to regulate nuclear gene expression first emerged from studies on the albostrians mutant of barley ${ }^{13}$. The expression of several chloroplast proteins encoded in the nucleus was reduced in mutants that had undifferentiated chloroplasts lacking ribosomes and were highly sensitive to photo-induced damage. Thus far, several chloroplastto-nucleus retrograde signaling pathways have been identified ${ }^{10-12}$. The intermediates of tetrapyrrole biosynthesis have been implicated in the communication between the chloroplast and nucleus in both Chlamydomonas and higher plants ${ }^{14,15}$. The application of inhibitors of tetrapyrrole biosynthesis and of intermediates in tetrapyrrole biosynthesis can lead to the repression and activation of nuclearencoded genes, respectively ${ }^{16}$. Further evidence was obtained through identification and characterization of gun (genome uncoupled) mutants (gun1 through gun5) that block the chloroplast-tonucleus retrograde signaling ${ }^{17}$. GUN2, GUN3, GUN4 and GUN5 encode proteins that participate in the tetrapyrrole biosynthetic pathway ${ }^{15,18,19}$. GUN2, GUN3 and GUN5 encode the haem oxygenase, the phytochromobilin synthase and the $\mathrm{H}$ subunit of $\mathrm{Mg}$ chelatase, respectively, in tetrapyrrole biosynthesis, whereas GUN4 encodes a chloroplast-localized protein that binds Proto/MgProto and regulates $\mathrm{Mg}$-chelatase activity. GUN5 has also been implicated as a receptor for ABA and antagonizes a group of WRKY transcription factors to relieve the inhibition of ABA response genes, such as $A B I 5, A B I 4$ and so on ${ }^{20,21}$. Although there are conflicting reports that the steady-state levels of Mg-protoporphyrin are not correlated to Lhcb (encoding a light-harvesting chlorophyll a/b-binding protein) expression levels ${ }^{22,23}$, the possibility exists that the rates of production and degradation of Mg-protoporphyrin function as a signal. In addition, impaired tetrapyrrole biosynthesis may cause localized generation of reactive oxygen species (ROS) or changes in the redox status of the chloroplast, which might trigger the signals ${ }^{22}$. The signals derived from the photosynthetic electron transfer (PET) chain seem to affect both photosynthesis-related and stress-related genes ${ }^{7-9}$. The redox state of the plastoquinone pool, other components of the photosynthetic electron transport chain and ROS are involved in the PET-derived signaling $7,8,24-27$. The signal is also induced by inhibition of photosynthetic gene expression (PGE) $)^{7,8,16}$.

Because the gun 1 mutant displays altered response to the PETdependent, tetrapyrrole-mediated and PGE-mediated signaling pathways, it seems that multiple signal transduction pathways are integrated within the chloroplast upstream of GUN1. GUN1, a chloroplastic pentatricopeptide repeat protein co-localized with the nucleoid ${ }^{28}$, may either generate or transmit a second, common signal to the nucleus. The nuclear-localized APETALA2 (AP2)-type transcription factor, ABI4, was proposed to inhibit $L h c b$ expression by competitively binding to the G-box so as to prevent the binding of G-box transcription factors in response to GUN1-derived chloroplast signals, thus mediating the previously proposed 'master switch $^{28}$. However, the molecular mechanism that relays the chloroplast signals through the cytosol to the nucleus remains unknown.

In this work, we show that a chloroplast envelope-bound plant homeodomain (PHD) transcription factor, designated PTM (for PHD type transcription factor with transmembrane domains), is activated by proteolytic cleavage and transmits multiple retrograde chloroplast signals to the nucleus.

\section{Results}

Functions of PTM in multiple retrograde signaling pathways. The simplified signal transduction pathway raises the key question of how chloroplast signals are transmitted to the nuclear-localized $\mathrm{ABI} 4$ to repress nuclear gene expression. A challenging task for understanding chloroplast-to-nucleus retrograde signaling is to identify the component for transducing chloroplast signals to the nucleus. Membrane-bound transcription factors (MTFs) have been shown to regulate diverse cellular functions through an intriguing regulated proteolytic activation mechanism ${ }^{29-31}$. These MTF proteins are associated with the intracellular membranes in their dormant forms. On stimulation, they are released from the membranes through proteolytic cleavage and its cleaved MTF fragments containing the transcription factor domain are redistributed to the nucleus, where they regulate the expression of particular nuclear genes $^{29-31}$. The At5g35210 gene encoding a putative chloroplast envelope-bound transcription factor $\mathrm{PTM}^{31}$ was chosen to test its function in mediating chloroplast signals to regulate photosynthetic nuclear gene expression. To investigate the PTM functions, we analysed the retrograde response in the Arabidopsis ptm mutant carrying a transfer-DNA (T-DNA) insertion in the second exon of PTM (Supplementary Fig. S1a-c). Under normal growth conditions, the ptm mutant plants were indistinguishable from wild type (Supplementary Fig. S1a). The expression level of $L h c b$ in wildtype and PTM/ptm seedlings decreased by about $97 \%$ following treatment with norflurazon (NF), an inhibitor of carotenoid biosynthesis, which causes photooxidative stresses within the chloroplast in the light (Fig. 1a). However, treatment of ptm, gun1 and abi4 with NF resulted in a reduction in Lhcb levels to about $13 \%, 22 \%$, and $15 \%$ those of the controls, respectively. NF treatment has also been shown to cause the disappearance of chloroplast ribosomes, which may indirectly trigger PGE pathway ${ }^{7}$. Because the signaling affected by chloroplast protein synthesis inhibitors has been shown to operate in 3 -day-old seedlings ${ }^{16}$, we treated older seedlings with NF to exclude the potential effects of PGE. The ptm seedlings, similar to the gun 1 and abi4 seedlings, were unable to repress $L h c b$ expression when treated with NF (Fig. 1b), which suggests that PTM has a role in the tetrapyrrole-mediated pathway. Treatment with plastid protein synthesis inhibitor lincomycin (Lin) also resulted in a reduction in Lhcb transcript levels, whereas the ptm seedlings, similar to the abi4 and gun1 seedlings, showed an increased accumulation of $L h c b$ compared with wild type (Fig. 1c). As NF and Lin treatments were performed in the light, it would be expected that ROS accumulate. To exclude the effects of ROS, we examined the Lhcb levels of the ptm mutant in the presence of Lin in the dark and our results showed that ptm also displayed a gun phenotype (Supplementary Fig. S2a), suggesting that PTM is involved in a PGE-dependent signaling pathway.

We then tested whether PTM is also involved in the PET-derived signaling. Treatment with high light (HL) causes a reduction of photosynthetic electron transport chain activity and produces ROS ${ }^{7,8}$. High levels of light were effective in preventing the expression of Lhcb in wild-type seedlings, whereas decreased inhibitory effects on the expression of Lhcb were observed in ptm, gunl and abi4 (Fig. 1d). We next examined the expression of APX2 (ascorbate peroxidase 2) upon exposure to HL (Fig. 1d and Supplementary Fig. S2b). Treatment with HL resulted in a rapid, transient accumulation of APX2 and high levels of APX2 were observed within 
a

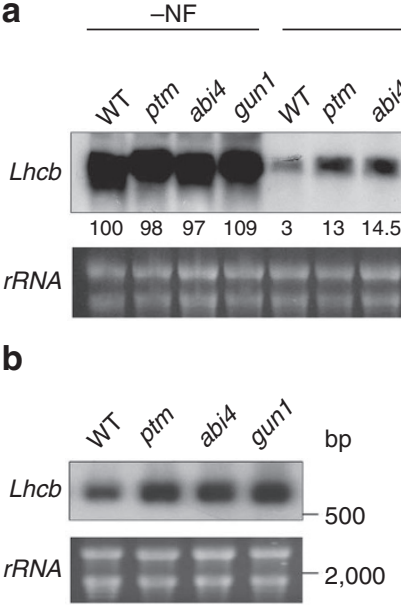

$+\mathrm{NF}$

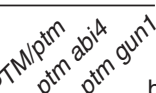

bp

C

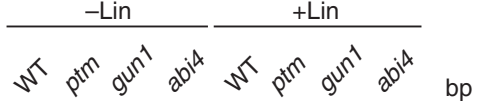

Lhcb
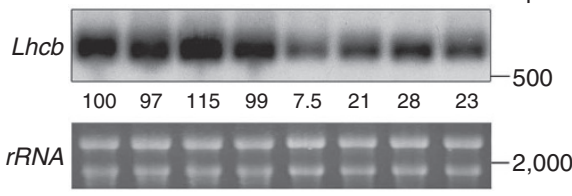

d

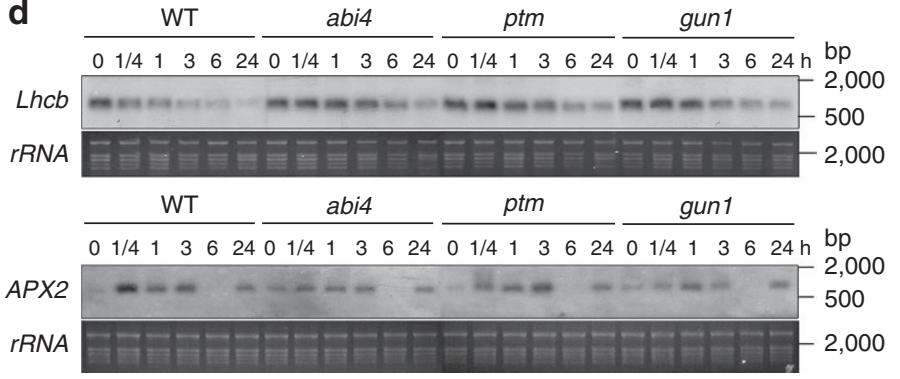

Figure 1 | The ptm mutant is a gun mutant. (a) Lhcb mRNA levels in seedlings of wild-type (WT), ptm, abi4, gun1, PTM/ptm (ptm complemented with PTM), ptm abi4 and ptm gun1 mutants after treatment with norflurazon (NF). Arabidopsis seedlings were grown on $1 / 2$ LS medium with $5 \mu M$ NF in the dark for 4 days and then transferred to light for 3 days. RNA was isolated, and Lhcb mRNA levels were determined by RNA gel blot analysis. rRNA, ethidium bromide-stained ribosome RNA. (b) Lhcb mRNA levels in 4-day-old green seedlings of WT, abi4, ptm and gun1 mutant seedlings after treatment with NF. Four-day-old Arabidopsis seedlings grown on 1/2 LS medium were treated with $5 \mu \mathrm{M}$ NF and collected 9 days later. (c) Lhcb mRNA levels of 6-day-old seedlings of WT, ptm, abi4 and gun1 mutants after treatment with lincomycin (Lin). Arabidopsis seedlings were grown on 1/2 LS medium with $220 \mu \mathrm{g}$ ml ${ }^{-1}$ Lin for 6 days. (d) Lhcb and APX2 mRNA levels in 4-week-old WT, abi4, ptm and gun1 mutant seedlings after HL treatment. Four-week-old WT, abi4, ptm and gun 1 mutant seedlings grown under normal light were exposed to $\mathrm{HL}$ intensities for the indicated times. These experiments (a-d) were repeated three times independently, and similar results were obtained. The results from a representative experiment are shown.

$15 \mathrm{~min}$ and maintained up to $3 \mathrm{~h}$ in wild type. The maximal level of APX2 was observed after 1 or $3 \mathrm{~h}$ in gun 1 , abi4 and ptm. HL is also known to be a rather complex signal that generates ROS and reduces plastoquinone pool, which may affect nuclear gene expres$\operatorname{sion}^{7,8}$. To distinguish these possibilities, we have examined the levels of Lhcb after treatments with dibromothymoquinone (DBMIB) (which reduce the plastiquinone pool), paraquat and rose Bengal (which would induce peroxides and singlet oxygen, respectively) under normal light conditions. The mRNA levels of Lhcb were about 3- to 4-fold higher in the ptm and gun1 mutants than wild-type plants (Supplementary Fig. S2c). These results suggest that PTM functions in multiple retrograde pathways.

Because the above results indicate that PTM, similar to GUN1 and ABI4, has a role in multiple chloroplast-derived signals to the nucleus, we investigated the relationship between PTM, GUN1 and ABI4. To this purpose, we crossed the single mutants and obtained gunl ptm and ptm abi4 double mutants (Supplementary Fig. S1d-f). Lhcb levels in gun1 ptm and abi4 ptm double mutants were similar to those in gun 1 and abi4 single mutants in the presence of NF, respectively (Fig. 1a). Analysis of Lhcb mRNA level in N-PTM/gun1 showed that the gun1 phenotype can be suppressed by constitutive expression of N-PTM during stress (Supplementary Fig. S2d), which is consistent with the action of PTM downstream of GUN1. No apparent difference in the Lhcb mRNA level was observed between wild-type and transgenic plants overexpressing N-PTM in the absence of inhibitors (Supplementary Fig. S2d).

Processing and subcellular localization of PTM during stress. The PTM protein of 1,706 amino acids has amino-terminal DDT (DNA-binding homeobox and different transcription factors) ${ }^{32}$ and $\mathrm{PHD}^{33}$ domains with highly conserved secondary and tertiary structures and four transmembrane domains at its carboxy-terminus (Supplementary Figs S3 and S4). Immunoblot analysis revealed the presence of one major band with a molecular mass of about $180 \mathrm{kDa}$, corresponding to the estimated full-length PTM, which was exclusively detected in the chloroplast outer envelope (Fig. 2a). Treatments with salts indicated that PTM was an intrinsic membrane protein (Supplementary Fig. S5a). PTM has a nuclear localization signal peptide in its N-terminus, according to the WoLF PSORT prediction ${ }^{34}$, and the generation of an N-terminal fragment lacking transmembrane domains but containing the complete DDT and PHD domains fused with green fluorescent protein (GFP) showed that the GFP signal was localized in the nucleus (Fig. 2b). Further chloroplast fractionation and immunoblot analysis showed that only one band, with a molecular weight of $\sim 58 \mathrm{kDa}$, was detected in the nuclear fraction (Fig. 2c). The immunoblot analysis with the antibody against the C-terminus of PTM showed that the processed form of PTM, C-PTM, was not detectable in the nucleus (Supplementary Fig. S5b).

It is likely that PTM is localized in the chloroplast envelope but released from the membrane by an as-yet-unidentified protease, with its cleaved MTF fragment containing the transcription factor domains being redistributed to the nucleus, where it regulates the expression of particular nuclear genes. To test this possibility, we monitored the levels of full-length PTM and its processed form after plants had been subjected to various treatments (Fig. 2d,e). The levels of full-length PTM increased during 4 days and decreased at day 5 after treatments with NF and Lin, and the PTM contents increased during the course of treatments with HL (Fig. 2d,e and Supplementary Fig. S5c). Treatments with HL, Lin and NF induced the detection of a lower band of $\sim 58 \mathrm{kDa}$, which was the same size as the $\mathrm{N}$-terminal fragment without transmembrane domains $(\mathrm{N}$ PTM; Fig. 2d,e). This may in fact be the processed form of PTM, as has been reported for other $\mathrm{MTFs}^{29-31}$. In addition, immunofluorescence analysis with anti-N-PTM antibody showed that the immunofluorescence signals were predominantly detected in chloroplasts before treatment (Fig. 2f). After NF and Lin treatments, the shift in colour of nucleus in merged images was most obvious (pale blue with inhibitors versus dark blue in control), probably as a result of migration of processed N-terminal PTM from the chloroplast to the 
a

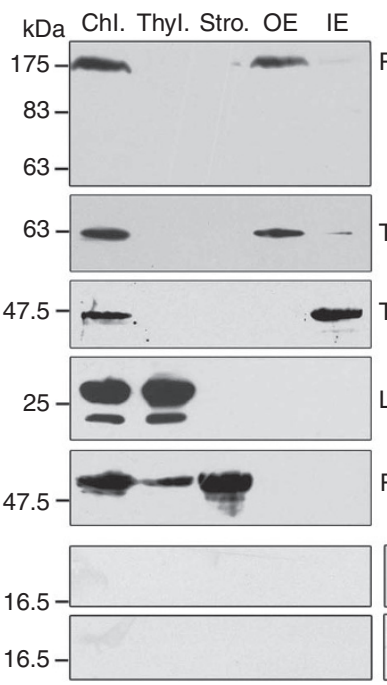

c

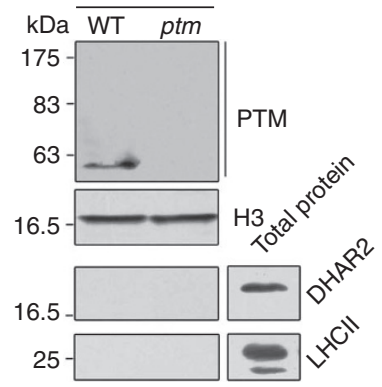

b

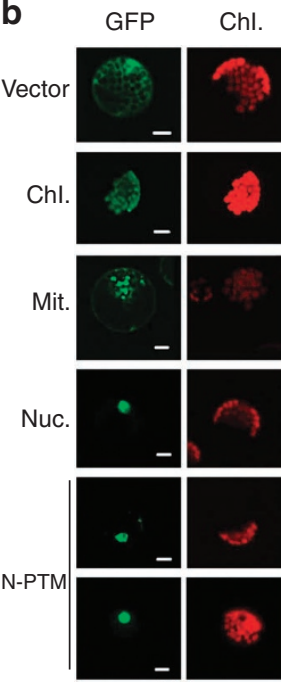

Bright field Merged

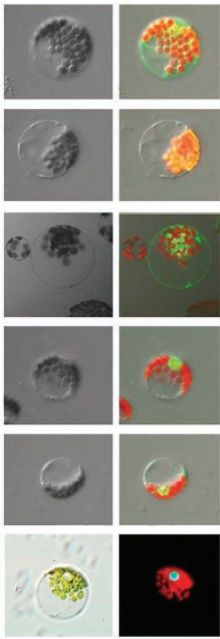

f

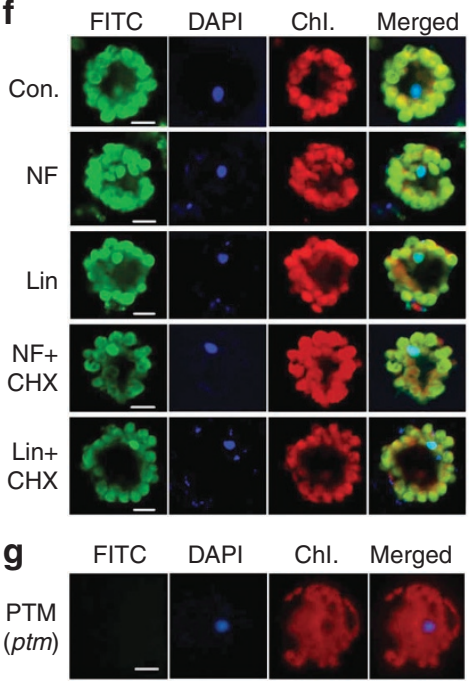

d

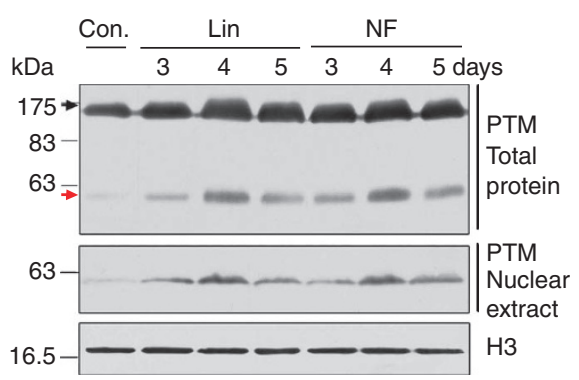

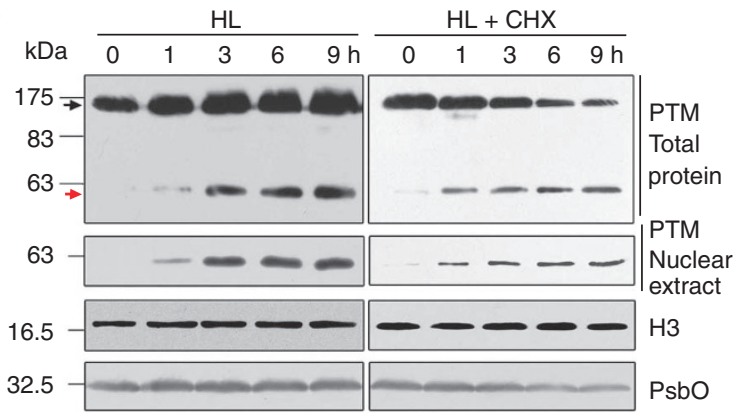

Figure 2 | Dual localization of PTM to the chloroplast and the nucleus. (a) Immunolocalization analysis of PTM. Intact chloroplasts (ChI.) were separated into stroma (Stro.), thylakoid membrane (Thyl.), outer envelope (OE) and inner envelope (IE) fractions. Polyclonal antibodies were used against RbcL, LHCII, TOC75, TIC40, DHAR2, Histone 3 and PTM. Sample loading was standardized by protein content ( $5 \mu \mathrm{g})$. (b) Subcellular localization of N-PTM by GFP assays. The constructs used for transformation are indicated to the left: Vector, signal of 35S::GFP vector; Chl., signal of chloroplast localization protein, RbcL; Nuc., signal of nuclear localization protein, fibrillarin; Mit., signal of mitochondrial localization protein, FRO1; N-PTM, signal from the NPTM-GFP fusion protein. A representative image from a single protoplast is shown. Scale bar, $10 \mu \mathrm{m}$. (c) Nuclear localization of the processed form of PTM. The nuclear extracts from wild-type (WT) and ptm plants were resolved on SDS-polyacrylamide gel electrophoresis (PAGE) followed by immunoblot analysis. (d) Effects of NF and Lin on PTM processing. Total proteins $(30 \mu \mathrm{g})$ were isolated from the seedlings after treatments with NF and Lin, separated by SDS-PAGE and immunodetected with anti-PTM. The black arrows indicate the full-length PTM protein, and the red arrows indicate the processed form of full-length PTM. (e) Effects of high light ( $\mathrm{HL}$ ) on PTM processing. Total proteins $(30 \mu \mathrm{g})$ were isolated from the HL-treated leaves in the presence $(\mathrm{HL}+\mathrm{CHX})$ or absence $(\mathrm{HL})$ of cycloheximide, and subjected to immunoblot analysis. (f) After treatments with Lin and NF in the presence or absence of cycloheximide (CHX), the protoplasts were incubated with anti-PTM antibody followed by incubation with a secondary antibody conjugated to FITC. An untreated sample was used as a control. Scale bar, $10 \mu \mathrm{m}$. (g) Immunofluorescence analysis of the ptm mutant with anti-PTM antibody. The 4,6-diamidino2-phenylindole (DAPI) fluorescence, shown in blue, indicates the location of the nucleus. Chlorophyll autofluorescence was detected and shown in red. Scale bar, $10 \mu \mathrm{m}$. These assays ( $\mathbf{a}-\mathbf{g}$ ) were repeated three times, and the results from a representative experiment are shown.

nucleus (Fig. 2f). A control experiment with the ptm mutant indicates the specificity of fluorescence signals (Fig. $2 \mathrm{~g}$ ). The increase in PTM levels observed in the immunoblot analysis after NF and Lin treatments was consistent with the observations that $\beta$-glucuronidase (GUS) levels and GUS activities increased after treatments with NF, and Lin in transgenic plants carrying a translational gene fusion construct PTM-GUS (Fig. 3a-f).

To examine whether N-PTM was derived from full-length PTM, the leaves were treated with cycloheximide (CHX) under HL. The nuclear-encoded PsbO protein levels were decreased gradually in the presence of $\mathrm{CHX}$, but remained almost unchanged in the absence of CHX (Fig. 2e), which indicates that CHX can efficiently inhibit nuclear-encoded protein synthesis. The full-length PTM protein contents were decreased in the presence of CHX (Fig. 2e) because of reduced protein synthesis and continued PTM degradation. The levels of N-PTM increased gradually after HL treatment in the presence of CHX. Examination of the processing and localization of
PTM in the gun1 mutant showed that the levels of full-length PTM and processed N-PTM were decreased during stress (Supplementary Fig. S5c), which suggests that GUN1 is important for increased PTM expression during stress. In addition, immunofluorescence analysis with anti-N-PTM antibody showed that the immunofluorescence signals in the chloroplasts were decreased after treatments with NF and Lin in the presence of CHX, whereas the signals in the nucleus were increased (Fig. 2f). These results indicate that NPTM was processed from full-length PTM. As shown in Supplementary Figure S5d, PTM processing was dramatically suppressed by serine protease inhibitor pefabloc, but not by aspartic protease inhibitor pepstatin. Further examination of the cleavage of PTM in isolated chloroplasts treated with Lin showed that PTM contents were decreased gradually in the absence of pefabloc, but the levels of PTM remained relatively stable in the presence of pefabloc. These results indicated that PTM can be processed by the protease in the envelope (Supplementary Fig. S5e). 


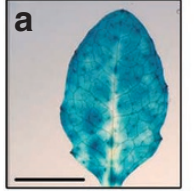

Control

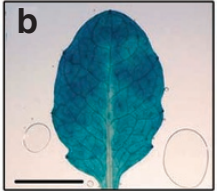

HL

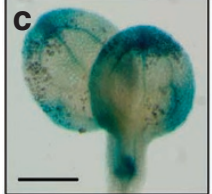

Control

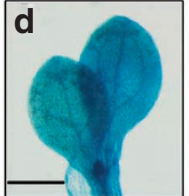

Lin

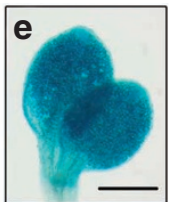

NF f

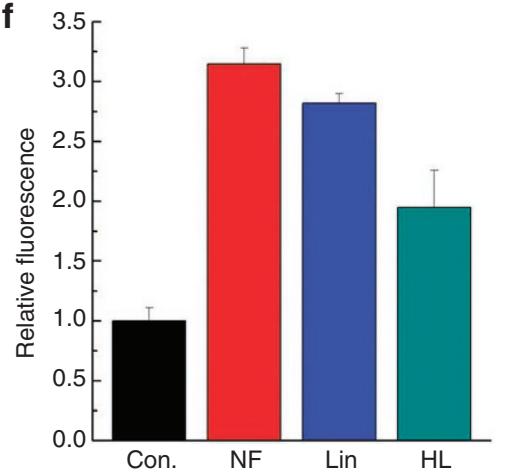

g

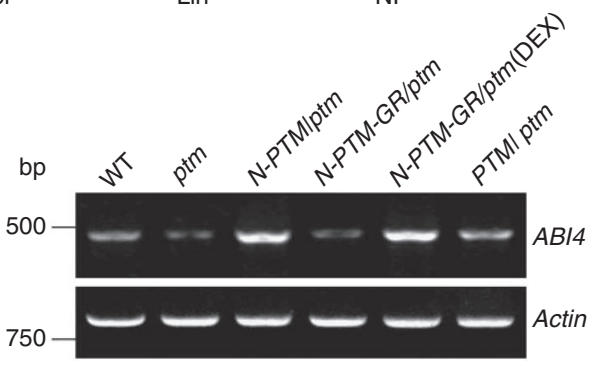

h

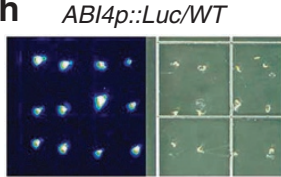

i $A B / 4 p:: L u c / p t m$

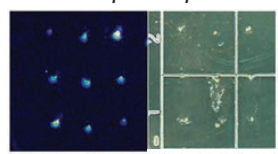

Figure 3 | Expression of PTMp::PTM-GUS and ABI4. (a) The GUS staining of the transgenic PTM-GUS leaves. Scale bar, $5 \mathrm{~mm}$. (b) The GUS staining of the transgenic PTM-GUS leaves after treatment with high light (HL; 1h). Scale bar, $5 \mathrm{~mm}$. (c) The GUS staining of the transgenic PTM-GUS seedling cotyledon. Scale bar, $0.5 \mathrm{~mm}$. (d,e) The GUS staining of the transgenic PTM-GUS seedling cotyledon after treatments with norflurazon (NF, 5 days) and lincomycin (Lin, 5 days), respectively. Scale bar, $0.5 \mathrm{~mm}$. (f) The GUS activities of the transgenic PTM-GUS seedling cotyledon after treatments with NF ( 5 days), $\mathrm{Lin}$ (5 days) or leaves after treatments with $\mathrm{HL}(1 \mathrm{~h})$. Values are means of triplicate measurements with error bars representing the standard deviation. (g) Expression of ABI4 in wild type (WT), ptm, N-PTM/ptm, PTM/ptm and N-PTM-GR/ptm. Six-day-old WT, ptm, PTM/ptm, N-PTM/ptm and N-PTM-GR/ptm seedlings were grown in 1/2 LS media used for RT-PCR (dexamethasone (DEX) indicates seedlings were grown in 1/2 LS media with $1 \mu M$ DEX). PCR amplification was performed using $A B / 4$ primers. Actin was used as a control. (h,i) Luminescence in the transgenic $A B / 4 p:: L u c / W T$ (h) and $A B / 4 p:: L u c / p t m$ (i) lines. These experiments ( $\mathbf{a}-\mathbf{e}$ and $\mathbf{g}-\mathbf{i}$ ) were repeated three times independently, and similar results were obtained. The results from a representative experiment are shown.

Transcriptional activation activity of PTM. Semi-quantitative reverse transcription PCR (qRT-PCR) analysis showed that $A B I 4$ expression was reduced in the ptm mutant, and the level of $A B I 4$ was restored in the ptm-complemented line (Fig. $3 \mathrm{~g}$ ). The effect of PTM on ABI4 expression was further examined by crossing $\mathrm{ptm}$ with plants carrying the luciferase reporter gene fused to the $A B I 4$ promoter $(A B I 4 p:: L u c)$. The luminescence intensities in the $p t m$ seedlings were considerably lower than those in wild type (Fig. 3h,i). These results indicate that PTM is required for the proper expression of $A B I 4$. We further generated transgenic plants expressing N-PTM proteins fused with a glucocorticoid receptor (GR) to control nuclear localization and our results showed that the expression of $A B I 4$ was recovered in N-PTM-GR/ptm only in the presence of $1 \mu \mathrm{M}$ dexamethasone but not in its absence (Fig. $3 \mathrm{~g}$ ). These results indicate that the nuclear localization of PTM is required for normal $A B I 4$ expression. Next we examined whether the ptm mutant showed ABA resistant. Our results showed that gun 1 and ptm appeared intermediate between wild type and abi4 in germination assays, which suggests the possible function of PTM in ABA signaling (Supplementary Fig. S6).

We next performed a chromatin immunoprecipitation (ChIP) assay to examine whether processed PTM binds to the promoter region of $A B I 4$. Multiplex PCR revealed that fragment $A B I 4 p-4$ $(-288 /-20)$ was enriched in the $A B I 4$ promoter in the anti-PTM samples compared with the ChIP samples prepared with preimmune antisera and actin controls (Fig. 4a,b). We next used a yeast one-hybrid assay to delineate the DNA sequences to which N-PTM binds. Gal4 transcriptional activation domain N-PTM (GAD$\mathrm{N}-\mathrm{PTM}$ ) fusion proteins, but not GAD alone, activated the $\mathrm{LacZ}$ reporter genes driven by fragment $A B I 4 p-4$, but not fragments $A B I 4$ 1-3 of $A B I 4$ promoters, which provided further support for these findings (Fig. 4c). These results suggest that PTM is associated with the ABI4 promoter in vivo.

Domain deletion analysis revealed that the portion of the $\mathrm{N}$ terminal fragments containing PHD domains are necessary and sufficient for activating $L a c Z$ reporter genes driven by the $A B I 4$ promoter (Fig. 4d,e). To test whether PTM has an intrinsic transcriptional regulatory activity, we fused PTM and N-PTM (the $\mathrm{N}$-terminus, containing complete DDT and PHD domains) with Gal4 DNA-binding domain. The pGBK-N-PTM fusion protein, but not the pGBK-PTM, activated transcription of reporter genes HIS3 and MEL1, which were driven by the Gal4 operator (Fig. 5a). Immunoblot analysis showed the expression of N-PTM, N-PTM mutants and PTM in the nucleus of yeast (Fig. 5b,c). This result suggests that the processing of PTM is necessary for its function. In addition, mutations of critical cysteine residues at positions 426 and 429 to alanines in the pGBK-N-PTM fusion protein failed to activate transcription of reporter genes HIS3 and MEL1 (Fig. 5a). Indeed, mutation analysis has also revealed the importance of corresponding cysteine residues in PHD domain for transcriptional activity $^{33,35}$, and substitution of cysteine residue with alanine in the PHD core region N-PTM (C429A) reduced the transcriptional activity, however, substitutions of other cysteine or histidine residues with alanine (N-PTM (C414A), N-PTM (C417A), N-PTM (C426A), N-PTM (H434A), N-PTM (H434A, C437A) and N-PTM (C452A, C455A)) had no effect. These results indicate that the $426 \mathrm{C}$ and $429 \mathrm{C}$ residues are necessary for transcription activation. In addition, PTM, but not C426A/C429A mutant protein, activated a luciferase reporter gene driven by the ABI4 promoter in Arabidopsis protoplasts (Fig. 5d). These results suggest that the PHD domain is essential for transcriptional activation activity and that C426 and C429 residues are important for PTM function. 


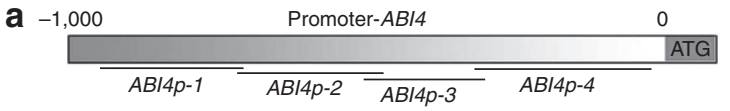

b

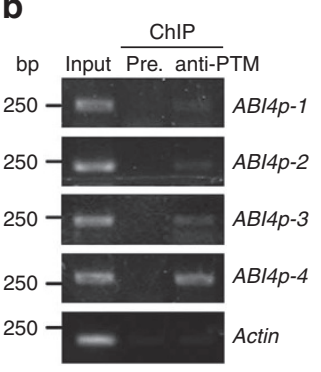

C

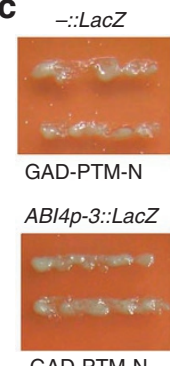

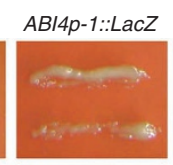

GAD-PTM-N

ABI4p-4::LacZ

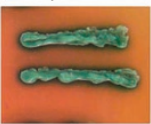

GAD-PTM-N

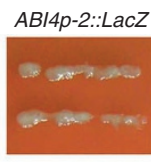

GAD-PTM-N
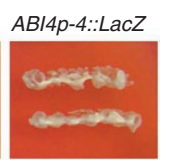

GAD d

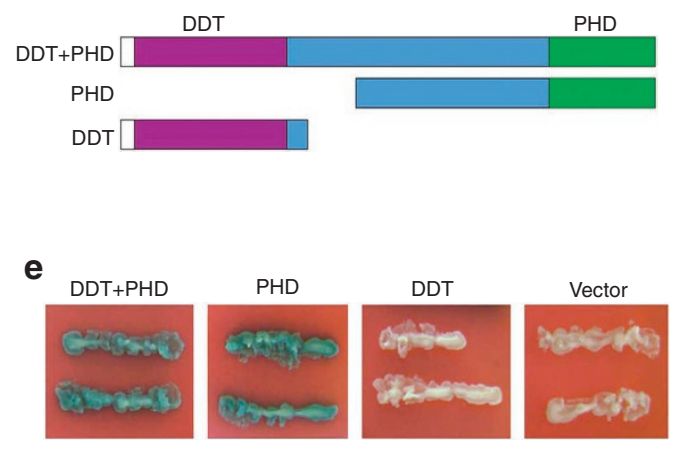

Figure 4 | PTM binds directly to the promoter of $\mathbf{A B I 4 . ~ ( a ) ~ D i a g r a m ~ o f ~ t h e ~ p r o m o t e r ~ f r a g m e n t s ~ o f ~} A B / 4$. The locations of PCR primers ( $A B / 4 p-1, A B / 4 p-2$, $A B / 4 p-3$ and $A B / 4 p-4)$ used for the enrichment test are indicated, and the numbers indicate base pairs relative to the initiation site, which is indicated by ' $O$ '. (b) PTM binds directly to AB/4 promoter. ChIP analysis was performed using N-PTM antibody, and genomic DNA obtained by ChIP was subjected to PCR analysis. Immunoprecipitation with a preimmune (Pre.) sample was used as a control. The PCR fragments $A B I 4 p-1, A B I 4 p-2, A B I 4 p-3$ and $A B I 4 p-4$ represent four fragments of $A B I 4$ promoter, which were shown in a. (c) PTM can activate expression of the $L a c Z$ reporter gene driven by the $A B I 4 p-4:$ : LacZ in yeast. The plasmids GAD-N-PTM with -::LacZ, ABI4p-1::LacZ, ABI4p-2::LacZ, ABI4p-3::LacZ and ABI4p-4::LacZ; and GAD itself, with ABI4p-4::LacZ, were co-transformed into the yeast strain EGY48. Transformants were grown on proper dropout plates containing X-gal (5-bromo-4-chloro-3-indolyl$\beta$-D-galactopyranoside) for blue colour development. (d) Diagram of PTM constructs (all fused with GAD) used in the yeast one-hybrid assay. The N-terminal PHD finger motif and the DDT domain are shown. (e) Yeast one-hybrid assay showing that the N-terminal fragments of PTM (containing the

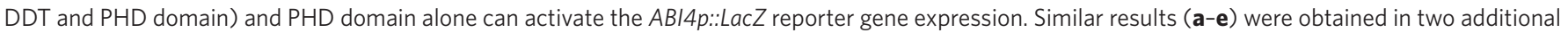
independent experiments.

PTM binding and histone modification during stress. A subset of PHD domains, including ING2 and ORC1, have been shown to interact with methylated histones ${ }^{35,36}$. Mutational analyses of human ING2 and Arabidopsis ORC1b PHD motif have demonstrated that residue W238 or G188 is part of the cage that contributes to the interaction with $\mathrm{H} 3 \mathrm{~K} 4 \mathrm{me} 3^{35-37}$. Residues D412, G421 and L423 of PTM are highly conserved in the equivalent positions (Fig. 6a). To investigate the functional relevance of the PHD domain of PTM, we first assessed its interaction with biotinylated histones. PTM was able to pull-down H3K4me3, whereas binding of PTM to unmodified histones $\mathrm{H} 3, \mathrm{H} 3 \mathrm{~K} 9 \mathrm{me} 3$ or $\mathrm{H} 4 \mathrm{~K} 20 \mathrm{me} 3$ was not detectable (Fig. 6b). Mutations of two cysteine residues of PTM-PHD to alanines (C426A, C429A) reduced, but did not completely abolish, the interaction of PTM-PHD with H3K4me3. Mutations of the D412, G421 and L423 residues of PTM revealed their importance for $\mathrm{H} 3 \mathrm{~K} 4 \mathrm{me} 3$ binding, although residual binding was still detectable (Fig. 6b).

The histone methylation and acetylation status were associated with gene transcriptional activity ${ }^{35-39}$. ChIP assays showed that H3K4me3 increased in fragment ABI4p-4 (-288/-20) of the ABI4 promoter when the plants were treated with NF, Lin or HL. Further examination of the acetylation status of PTM-bound region of ABI4 promoter revealed that $\mathrm{H} 3 \mathrm{~K} 9,14$ acetylation increased after treatments with NF, Lin or HL (Fig. 6c). However, HL-induced increase in $\mathrm{H} 3 \mathrm{~K} 4 \mathrm{me} 3$ and $\mathrm{H} 3 \mathrm{~K} 9,14 \mathrm{ac}$ was retained in ptm, which suggests that PTM is not required for histone modifications (Fig. 6d).

PTM activation associated with $A B I 4$ and $L h c b$ gene expression. We next monitored the ABI4 and PTM expression during retrograde responses by transforming Arabidopsis plants with ABI4p:: Luc and PTMp::Luc. The activities of PTMp::Luc showed a very similar pattern to that of $A B I 4 p:: L u c$, and the LUC activities of both PTMp::Luc and ABI4p::Luc were increased after HL treatment (Supplementary Fig. S7a,b). The LUC activities of both PTMp::Luc and $A B I 4 p:: L u c$ increased with the duration of treatments up to 4 days, and LUC activities decreased after treatment for 5 days in the presence of Lin or NF (Supplementary Fig. S7c,d). To determine whether the reporter gene expression was the causation of a change in mRNA levels, we examined the levels of both ABI4 and PTM with qRT-PCR. Similar results to the changes of LUC activities in response to retrograde signal were obtained (compare Fig. 7a,b with Supplementary Fig. S7a,b; compare Fig. 7c,d with Supplementary Fig. S7c,d). Genevestigator microarray data also showed that ABI4 expression is induced by environmental stresses, such as high temperature, HL, cold, salt stress and so on ${ }^{40}$. These results indicate that induction of $A B I 4$ expression may be important for its function in chloroplast-to-nucleus signaling.

We further elucidated the role of PTM processing in regulating Lhcb expression in response to retrograde signals under HL by treating leaves with protease inhibitor to block PTM processing. Under HL, qRT-PCR analysis showed that the level of ABI4 was increased by about 14 - and 4 -fold, respectively, in the absence and presence of protease inhibitor pefabloc (Fig. 7e). Treatment with pepstatin had no inhibitory effect on ABI4 expression under HL (Fig. 7e). $L h c b$ level was decreased by about $90 \%$ after treatments with HL in the absence of protease inhibitors or in the presence of pepstain. However, the level of $L h c b$ was about $40 \%$ of the controls after HL treatment in the presence of pefabloc (Fig. 7f). Similar results to the changes of LUC activities in response to retrograde signals during HL treatment were obtained (compare Fig. 7e,f with Supplementary Fig. S7e,f). These results suggest that the processing of PTM is important for mediating chloroplast signals to the nucleus.

\section{Discussion}

We have demonstrated here for the first time, to our knowledge, that regulated intramembrane proteolysis is involved in regulating retrograde chloroplast-to-nucleus signaling. The dormant PTM is associated with chloroplast envelope, while only processed form of PTM was detected in nucleus, where it activates $A B I 4$ transcription in a PHD-dependent manner (Fig. 8). Transcriptional regulation of gene expression depends on the reading of histone post-translational modifications ${ }^{35}$. The PHD finger has been identified as a bona fide 'reader' domain because PHD fingers recognize histone post-translational modifications. Our results show that PTM contains 

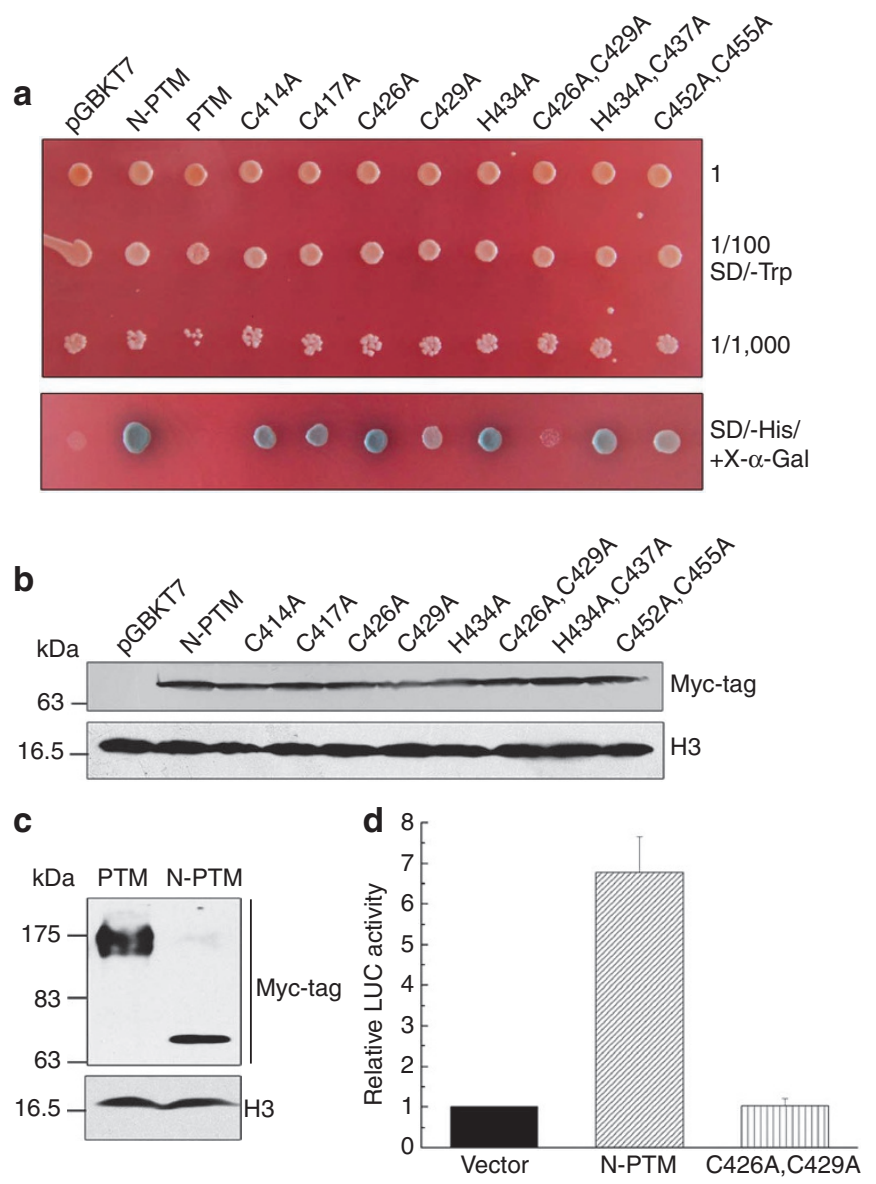

Figure 5 | PTM has an intrinsic transcription activation activity.

(a) pGBK-N-PTM, N-PTM (C414A), N-PTM (C417A), N-PTM (C426A), N-PTM (C429A), N-PTM (H434A), N-PTM (H434A, C437A) and N-PTM (C452A, C455A) fusion proteins activate HIS and MEL reporter gene expression in yeast $\mathrm{Y} 2 \mathrm{HG}$ old, whereas pGBKT7 alone, pGBK-PTM and N-PTM (C426A, C429A) fail to activate reporter gene expression. Similar results were obtained in two additional independent experiments. The results from a representative experiment are shown. (b) Immunoblot analysis of the N-PTM and N-PTM mutant proteins in the yeast nucleus. The nuclear proteins of yeast were isolated, separated by SDSpolyacrylamide gel electrophoresis, and immunodetected with anti-Myctag (the c-Myc epitope tag in the vector pGBKT7). The anti-H3 antibody was used as a control. (c) Immunoblot analysis with anti-Myc-tag showing the location of PTM and N-PTM in the yeast nucleus. (d) Wild-type pBSK35S::N-PTM, but not pBSK-35S::N-PTM (C426A, C429A) or pBSK-35S itself, activates $A B \mid 4 p::$ Luc reporter gene expression in Arabidopsis protoplasts. Error bars represent s.d. values of triplicate experiments. LUC, luciferase.

a functional PHD domain that interacts with $\mathrm{H} 3 \mathrm{~K} 4 \mathrm{me} 3$ (Fig. 6). The interaction between $\mathrm{H} 3 \mathrm{~K} 4 \mathrm{me} 3$ and proteins containing a PHD is ultimately coupled to changes in the histone acetylation and/or methylation statuses that are responsible for transcriptional regulation ${ }^{37-39}$. PTM-dependent transcription activation is associated with an increase in the acetylation of $\mathrm{H} 3 \mathrm{k} 9,14$ and trimethylation of H3K4 in response to treatments with NF, Lin and HL (Fig. 6). Thus, our results indicate that PTM is a $\mathrm{H} 3 \mathrm{~K} 4 \mathrm{me} 3$ effector protein and that activation of $A B I 4$ gene expression is mediated by binding of a functional domain to the $A B I 4$ promoter.

PTM seems to participate in multiple chloroplast signals because the expression of $L h c b$ was uncoupled during various treatments (Figs 1 and 8). ABI4 expression levels were elevated in response to treatments with Lin, NF and HL (Fig. 7). In addition, expression a

AtORC1b (168) D--CQICFK---SDTNIMIECDD----CLG-GFHLKCLKPPLKEVPEGDWICQFCEVKK HsING2 (215) Y--CL-CNQV SYGE-MIGCDNEQCPIEWFHFSCVSLTYKPKGKWY---CPKCRGDN PTM (412) DECRICGMDGT -..--LLCCDG-.--CPL-AYHSRCIGVVKMYIPDGPWFCPECTINKK
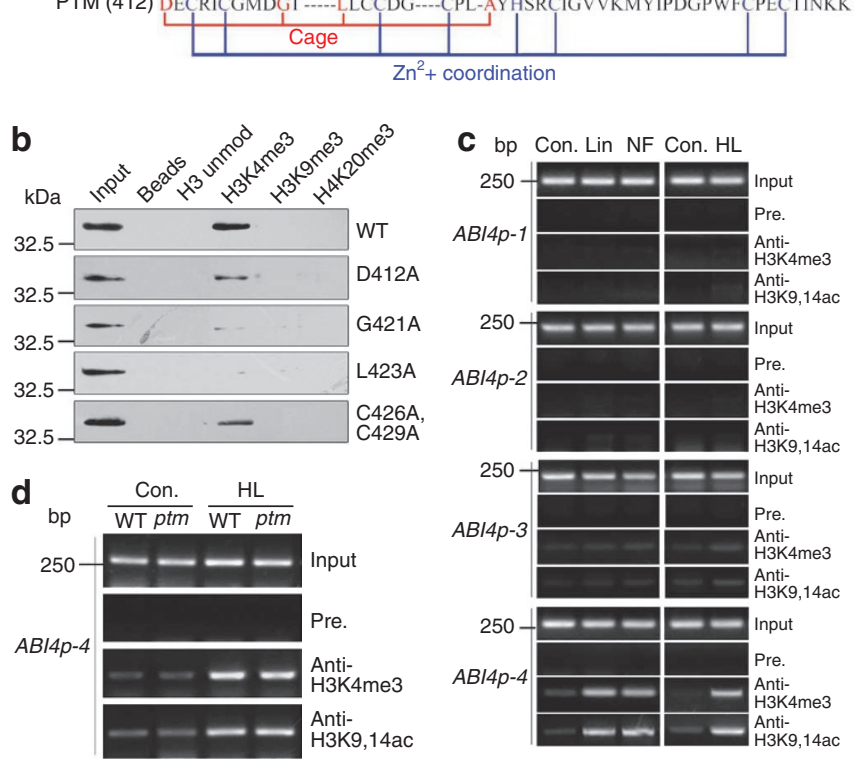

Figure 6 | Histone modifications in the promoter of $\boldsymbol{A B I 4}$. (a) The main cysteine $(\mathrm{C})$ and histidine $(\mathrm{H})$ residues of PHD regions appear in blue, and aspartic $(D)$, glycine $(G)$, leucine $(L)$ and alanine $(A)$ residues of the cage are in red. The key $\mathrm{C}$ and $\mathrm{H}$ residues of the $\mathrm{Zn}^{2+}$ fingers, and the $\mathrm{D}, \mathrm{G}, \mathrm{L}$ residues of the cage that were mutated in this study are shown. (b) PTM specifically interacts with $\mathrm{H} 3 \mathrm{~K} 4 \mathrm{me} 3$. Pull-down assays of biotinylated native $\mathrm{H} 3$ unmod., H3K4me3, H3K9me3 and H4K2Ome3 peptides by GST-PTM-PHD and various mutations of GST-PTM-PHD (D412A, G421A, L423A, C426A/C429A) are shown. Bound proteins were detected by immunoblot analysis with anti-GST. (c) The levels of H3K4me3 and $\mathrm{H} 3 \mathrm{~K} 9,14 \mathrm{ac}$ were increased in the promoter of $A B / 4$ after NF, LIN and $\mathrm{HL}$ treatments for $6 \mathrm{~h}$. ChIP assays were performed with anti-H3K $4 \mathrm{me} 3$ and anti-H3K9,14ac antibodies with Arabidopsis chromatin. The samples were analysed by PCR using primers that matched selected fragments of $A B I 4 p$ 1, $A B|4 p-2, A B| 4 p-3$ and $A B \mid 4 p-4$. The samples were treated as described in fig 1a,c,d. The untreated samples were used as control. (d) ChIP analyses of the levels of $\mathrm{H} 3 \mathrm{~K} 4 \mathrm{me} 3$ and $\mathrm{H} 3 \mathrm{~K} 9,14 \mathrm{ac}$ in the promoter of $\mathrm{AB} / 4$ after $\mathrm{HL}$ treatment. After wild-type (WT) and ptm mutant plants were treated by $\mathrm{HL}$ for $6 \mathrm{~h}, \mathrm{ChIP}$ assays were performed with anti-H3K $4 \mathrm{me} 3$ and antiH3K9,14ac antibodies with WT and ptm mutant chromatin. The samples were analysed by PCR using primers that matched selected fragment of $A B / 4 p-4$. The untreated samples were used as controls. These experiments (b-d) were repeated three times independently, and similar results were obtained. The results from a representative experiment are shown. Con. control; Pre., preimmune.

of PTM and ABI4 showed similar patterns during treatments with NF, Lin and HL (Fig. 7 and Supplementary Fig. S7). Treatment with $\mathrm{HL}$ in the presence of perfabloc, which inhibited the processing of PTM, not only lowered the increasing extent of $A B I 4$ expression but also resulted in the derepression of $L h c b$ expression (Fig. 7f). Taken together, these results demonstrate the role of PTM processing in the activation of $A B I 4$, which is important for the suppression of Lhcb during the retrograde signaling. Our results support a model for the critical function of PTM in mediating signals from damaged and undeveloped chloroplasts to the nucleus (Fig. 8). However, transgenic plants overexpressing the processed PTM did not induce a retrograde response in the absence of inhibitors (Supplementary Fig. S2d). Perhaps an additional signal mediated by GUN1 is also required. Woodson et al. ${ }^{41}$ reported that haem can act as a 

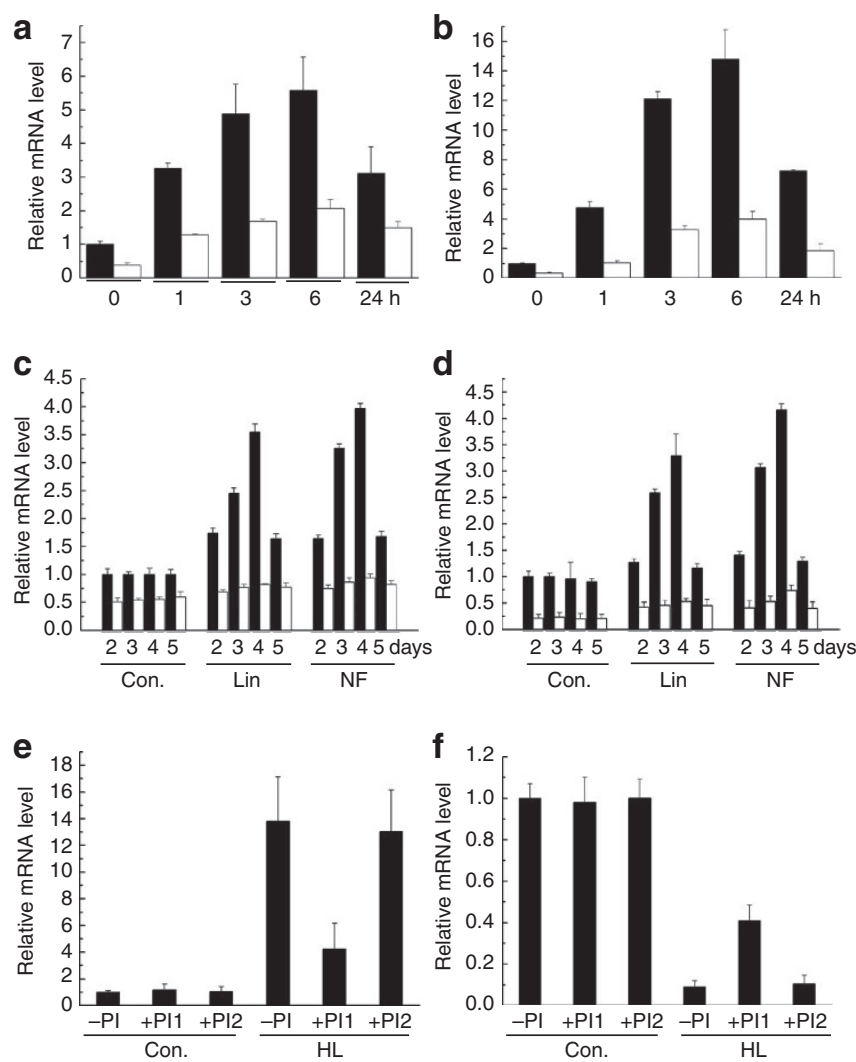

Figure 7 | Activation of $\boldsymbol{A B I 4}$ gene expression by PTM. (a) Expression of PTM in WT and gun1 after HL treatment. After treatment with HL for various numbers of hours, RNA was extracted and the level of PTM mRNA was analysed by qRT-PCR. Black and white bars indicate wild type (WT) and gun1, respectively. (b) Expression of ABI4 in WT and gun1 after HL treatment. After treatments with $\mathrm{HL}$ for various numbers of hours, RNA was extracted and the level of ABI4 mRNA was analysed by qRT-PCR. Black and white bars indicate WT and gun1, respectively. (c) Expression of PTM in WT and gun1 after treatments with NF and Lin. WT and gun1 mutant seedlings were grown in media in the presence of Lin and NF and collected after various numbers of days. After treatments, RNA was extracted and the level of PTM mRNA was analysed by qRT-PCR. Black and white bars indicate WT and gun1, respectively. (d) Expression of $A B / 4$ in WT and gun1 after treatments with NF and Lin. WT and gun1 seedlings were grown in media in the presence of Lin and NF, and collected after various numbers of days. After treatments, RNA was extracted and the level of $A B / 4$ mRNA was analysed by qRT-PCR. Black and white bars indicate WT and gun1, respectively. (e) Transcript levels of $A B / 4$ in the presence of protease inhibitors under $\mathrm{HL}$. After treatments with $\mathrm{HL}$ in the presence of pefabloc (+PI1) and pepstatin (+PI2) or absence of protease inhibitors ( $-I P$ ) for 6 h, RNA was extracted, and the level of ABI4 mRNA was analysed by qRT-PCR. The samples without $\mathrm{HL}$ treatment were used as control (Con.). (f) Transcript levels of $L h c b$ in the presence of protease inhibitors under $\mathrm{HL}$. After treatments with $\mathrm{HL}$ in the presence of pefabloc $(+\mathrm{PI} 1)$ and pepstatin (+PI2) or absence of protease inhibitors ( $-\mathrm{IP})$ for $6 \mathrm{~h}, \mathrm{RNA}$ was extracted and the level of AB/4 mRNA was analysed by qRT-PCR. The samples without $\mathrm{HL}$ treatment were used as control (Con.). Values are means of triplicate measurements with error bars representing the standard deviation.

positive signaling molecular regulating the photosynthetic-associated nuclear expression. Haem is likely to be responsible for the positive regulation of the photosynthetic-associated nuclear gene expression. There may be possible for a role of Proto/MgProto in this process. How tetrapyrroles may influence PTM and its ability to signal appears to be still an open question.

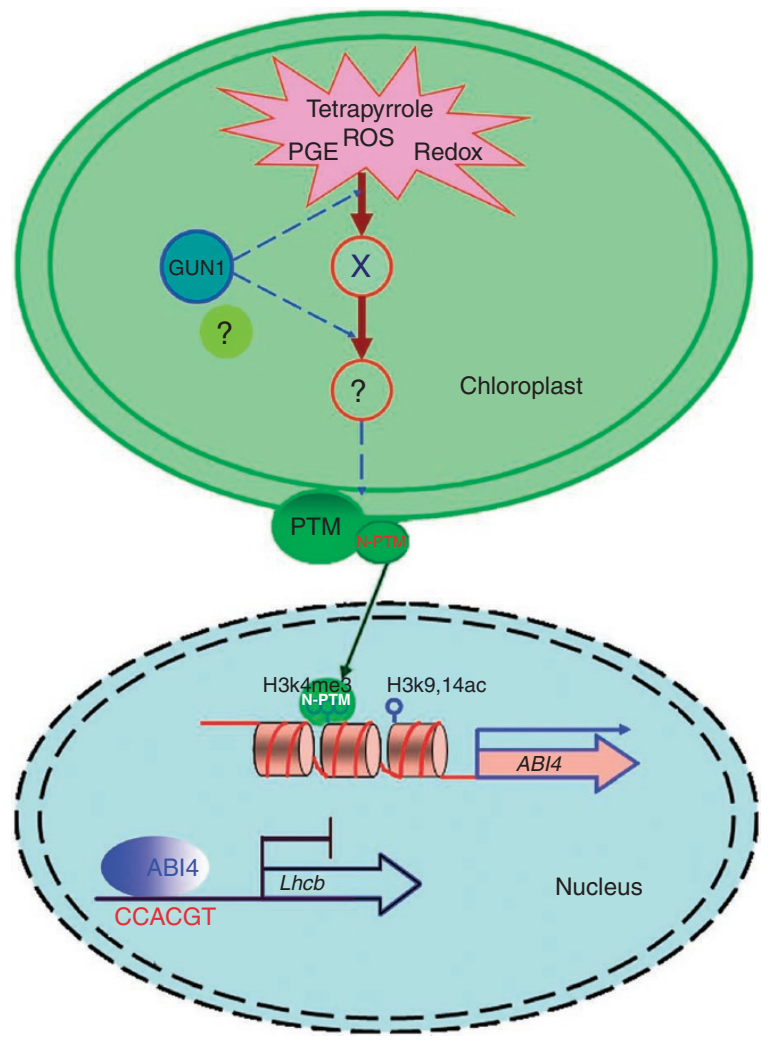

Figure 8 | A model showing that PTM acts as a sensor/transducer in the retrograde signaling pathways from chloroplasts. When plastid development is impaired or when plastids are stressed, inhibition of PGE, perturbation of tetrapyrrole biosynthesis, production of ROS and changes in the redox state of PET generate chloroplast retrograde signals. These signals converge on GUN1, which either generates or transmits a second signal. X could either be a process facilitated by GUN1 or a product of GUN1 activity. Unknown steps in the pathway are indicated by question marks. In response to chloroplast signals, PTM is processed into a mature form, which is redistributed to the nucleus, where it binds to the promoter of $A B / 4$. Activation of $A B / 4$ expression was associated with histone modifications.

These results provide a molecular basis for the critical function of PTM in mediating signals from chloroplasts to the nucleus and shed new light on the mechanism whereby the chloroplast signals are transmitted to the nucleus through the cytosol. Genes for PTM homologs exist in the nuclear genomes of plant species (Supplementary Fig. S3). All PTM proteins share conserved DDT and PHD domains at their N-terminus and four transmembrane domains at their C-terminus, which suggests an evolutionarily conserved mechanism for retrograde chloroplast signaling in higher plants. However, such mechanism is unlikely to exist in Chlamydomonas because no homologous protein to PTM was found. The chloroplast localization of PTM places it an ideal place to initiate rapid transcriptional responses to developmental and functional states of chloroplasts. As chloroplasts serve as the sensors of changes in growth environment, such communication could be important in establishing proper balance of gene expression allowing plants to respond to stressful environment. Future analysis regarding how PTM senses retrograde signals within the chloroplast, how dormant PTM transcription factor is activated, and how PTM works in this complex signaling network will be of great interest in understanding cell signaling in plants and will lead to a further understanding of the role of chloroplast retrograde signaling in plant growth and development. 


\section{Methods}

Plant materials and growth conditions. The following Arabidopsis thaliana mutants in the Columbia ecotype were obtained from the Arabidopsis Biological Resource Center: abi4 (abi4-1, an X-ray mutant (CS8104)), gun1 (SAIL_742_A11, a T-DNA insertion mutant) and ptm (SALK_013123, a T-DNA insertion mutant) The T-DNA insertion homozygous lines were confirmed by PCR using the following gene-specific and T-DNA-specific primers: PTMLP, PTMRP, and T-DNA $L B$ for PTM; and GUN1LP, GUN1RP, and T-DNA $L B$ for GUN1. The DNA fragments around the sites of T-DNA insertions were amplified and cloned into the plasmid vector pGEM-T (Promega), and the precise location of T-DNA insertion was determined by sequencing. Plants homozygous for the T-DNA insertions (identified by PCR analyses) were obtained, and single mutants were backcrossed to wild-type plants three times before generating double mutants to segregate out additional mutations.

All of the mutants and wild-type plants were grown in growth chambers at $22^{\circ} \mathrm{C}$ and $120 \mu \mathrm{mol} \mathrm{m}^{-2} \mathrm{~s}^{-1}$ with a 10 -h-light and 14-h-dark photoperiod. For NF and Lin treatments, the mutant and wild-type seeds were surface-sterilized and plated on 1/2 Linsmaier and Skoog (1/2 LS) medium (PhytoTechnology Laboratories, LLC) containing $2 \%$ sucrose and $0.8 \%$ agar with either $5 \mu \mathrm{M}$ NF (Sandoz Pharmaceuticals) or $220 \mu \mathrm{g} \mathrm{ml}^{-1}$ Lin (Sigma). For HL treatments, seedlings grown for 4 weeks under normal light conditions were exposed to $\mathrm{HL}\left(1,800 \mu \mathrm{mol} \mathrm{m}^{-2} \mathrm{~s}^{-1}\right)$ for various lengths of time.

Northern blots analysis. RNA was extracted with the RNeasy kit (Qiagen). For northern blot analysis, total RNA isolated from the wild-type and mutant plants was fractionated, transferred onto nylon membranes, and subsequently probed with ${ }^{32}$ P-labeled complementary DNA probes.

Semi-quantitative and quantitative RT-PCR. For semi-quantitative RT-PCR, total RNA was treated with RNase-free DNase I (Takara) to remove genomic DNA before first-strand cDNA synthesis. First-strand cDNA was generated using the PrimeScript II 1st Strand cDNA Synthesis Kit (Takara), and PCR analysis was performed with gene-specific primers using the first-strand cDNA as a template. For quantitative real-time RT-PCR, the RNA samples were reverse transcribed into first-strand cDNA using a PrimeScript RT reagent Kit (Takara). PCR was then carried out using gene-specific primers and SYBR Premix ExTaq reagent (Takara) with an Mx3000p Real-time PCR System (Agilent, Stratagene) following the manufacturer's instructions. PCR reactions were performed in triplicate for each sample, and the expression levels were normalized to an actin gene.

Yeast transcription activity. For the transcription activity assay, pGBKT7, pGBK-N-PTM, N-PTM(C426A,C429A), N-PTM(C414A), N-PTM(C417A), N-PTM(C426A), N-PTM(C429A), N-PTM(H434A), N-PTM(H434A,C437A) and $\mathrm{N}-\mathrm{PTM}(\mathrm{C} 452 \mathrm{~A}, \mathrm{C} 455 \mathrm{~A})$ were transformed into Y2HGold yeast cells and streaked on synthetic dropout nutrient medium (SD/-Trp) or (SD/-His) containing X- $\alpha-\mathrm{Gal}$ agar plates.

Yeast one-hybrid assay. The plasmid GAD-N-PTM, with -::LacZ (vector of pLacZi), ABI4p-1::LacZ, ABI4p-2::LacZ, ABI4p-3::LacZ, and ABI4p-4::LacZ, and GAD itself, with $A B I 4 p-4:: L a c Z$, were co-transformed into the yeast strain EGY48 using standard transformation techniques. For the domain deletion assay, the GAD-DDT, GAD-PHD and GAD-(DDT + PHD) of PTM were co-transformed with $A B I 4 p-4:: L a c Z$ into the yeast strain EGY48 using standard transformation techniques. Transformants were grown on proper dropout plates containing X-gal (5-bromo-4-chloro-3-indolyl- $\beta$-D-galactopyranoside) for blue colour development ${ }^{42}$.

Protoplast transient expression assay. The reporter construct $A B I 4 p:: L u c$, the effector plasmid and the 35S::GUS construct were co-transformed into protoplasts ${ }^{43}$. After transformation, the protoplasts were incubated under light for $12-15 \mathrm{~h}$. The protoplasts were pelleted and resuspended in $100 \mu \mathrm{l}$ of $1 \times$ CCLR buffer (Promega). For the luciferase activity assay, $5 \mu \mathrm{l}$ of the extract was mixed with $50 \mu \mathrm{l}$ of luciferase assay substrate (Promega), and the activity was detected with the Modulus luminometer/fluorometer with a luminescence kit. For the GUS enzymatic assay, $5 \mu \mathrm{l}$ of the extract was incubated with $50 \mu \mathrm{l} 4$-methylumbelliferyl $\beta$-D-glucuronide assay buffer $(50 \mathrm{mM}$ sodium phosphate, $\mathrm{pH}$ 7.0, $1 \mathrm{mM} 4$-methylumbelliferyl $\beta$-D-glucuronide, $10 \mathrm{mM}$ EDTA, $10 \mathrm{mM} \beta$-mercaptoethanol, $0.1 \%$ sarkosyl, $0.1 \%$ Triton X-100) at $37^{\circ} \mathrm{C}$ for $15 \mathrm{~min}$, and the reaction was stopped by adding $945 \mu \mathrm{l}$ of $0.2 \mathrm{M} \mathrm{Na}_{2} \mathrm{CO}_{3}$. The fluorescence was measured using a Modulus Luminometer/ Fluorometer with a ultraviolet fluorescence optical kit (Turner Biosystems). The reporter gene expression levels were expressed as relative LUC/GUS ratios.

Chromatin immunoprecipitation. For immunoprecipitation, $10 \mu \mathrm{l}$ of antiH3K4me3, anti-H3K9,14ac (Upstate Biotechnology), or anti-PTM antibodies (rabbit antiserum against the synthesized peptides, Supplementary Table S1) were incubated in phosphate-buffered saline solution with Protein G-agarose (Upstate Biotechnology), and then $1 \mathrm{mg}$ of chromatin protein extract was added. Finally, the purified DNA was diluted to $100 \mu$ with $0.5 \mu$ l used for each PCR assay ${ }^{44}$. PCR primers used in the ChIP assays are described in Supplementary Table S2. As negative controls, the ChIP experiments were performed using protein G-agarose with preimmune serum.

Chloroplast isolation and fractionation. The Arabidopsis leaves were homogenized in $330 \mathrm{mM}$ sorbitol, $20 \mathrm{mM}$ Tricin- $\mathrm{NaOH}$, pH 7.6, $5 \mathrm{mM}$ EGTA, $10 \mathrm{mM}$ $\mathrm{Na}_{2} \mathrm{CO}_{3}, 0.1 \%(\mathrm{w} / \mathrm{v})$ bovine serum albumin (BSA) and $330 \mathrm{mgl}^{-1}$ ascorbate, filtered and centrifuged for $5 \mathrm{~min}$ at $2,000 \mathrm{~g}$. The pellet was resuspended in $330 \mathrm{mM}$ sorbitol, $20 \mathrm{mM}$ HEPES-KOH, pH 7.6, $5 \mathrm{mM} \mathrm{MgCl}_{2}, 10 \mathrm{mM} \mathrm{Na}_{2} \mathrm{CO}_{3}, 0.1 \%(\mathrm{w} / \mathrm{v})$ BSA and $2.5 \mathrm{mM}$ EDTA, and intact chloroplasts were isolated though a $40 \% / 70 \%$ step Percoll gradient ${ }^{45}$. The intact chloroplasts were resuspended in hypertonic buffer ( $0.65 \mathrm{M}$ sucrose, $10 \mathrm{mM}$ Tricine- $\mathrm{KOH}, \mathrm{pH} 7.9)$, and the suspension was homogenized in a Dounce tissue grinder. Thylakoids were then pelleted at $4,500 \mathrm{~g}$ for $30 \mathrm{~min}$ at $4{ }^{\circ} \mathrm{C}$, and the crude envelopes were obtained after the supernatant was subjected to centrifugation at $200,000 \mathrm{~g}$ for $1 \mathrm{~h}$. The crude envelopes were resuspended in $1 \mathrm{ml} 0.4 \mathrm{M}$ sucrose, $10 \mathrm{mM}$ Tricine- $\mathrm{KOH}, \mathrm{pH} 7.9$, and then the suspension was concentrated through a discontinuous sucrose gradient $(1,0.8$ and $0.46 \mathrm{M}$ sucrose) at $120,000 \mathrm{~g}$ for $5 \mathrm{~h}$. After centrifugation, the inner envelope and outer envelope fractions were recovered separately from the gradient, and the envelopes were further purified on linear sucrose gradients $(0.59-1.2 \mathrm{M}, 10 \mathrm{mM}$ Tricine-KOH, pH 7.9) ${ }^{46}$.

Isolation of nuclear protein extracts. The nuclear protein extracts were isolated using the CelLytic PN Isolation/Extraction Kit (Sigma-Aldrich).

Pull-down assays. The pGST-PTM-PHD and the mutant proteins were expressed and purified using GST Bind Kits (Novagen) as described by the manufacturer's protocol. Biotinylated histone peptides ( $1 \mu \mathrm{g}$ each; Supplementary Table S1) were incubated with $1 \mu \mathrm{g}$ of purified pGST-PTM-PHD and the mutant proteins in binding buffer $(0.1 \mathrm{M}$ phosphate, $0.15 \mathrm{M} \mathrm{NaCl}, \mathrm{pH} 7.2)$ overnight at $4^{\circ} \mathrm{C}$. After a 1-h incubation with Pierce Streptavidin Agarose Resin (Thermo Scientific), beads were washed five times with the binding buffer, and bound proteins were analysed by immunoblotting using an anti-GST antibody (Santa Cruz Biotechnology).

Immunofluorescence microscopy. The wild-type leaf sections were washed three times in PBS and incubated for $6 \mathrm{~h}$ at room temperature in a cell-wall digestion solution preheated at $37^{\circ} \mathrm{C}(20 \mathrm{mM}$ MES, $\mathrm{pH} 5.7,1.5 \%(\mathrm{w} / \mathrm{v})$ cellulase $\mathrm{R} 10,0.4 \%$ (w/v) macerozyme R10, $0.4 \mathrm{M}$ mannitol, $20 \mathrm{mM} \mathrm{KCl}, 10 \mathrm{mM} \mathrm{CaCl} 2,5 \mathrm{mM} \beta$-mercaptoethanol, $0.1 \% \mathrm{BSA}, 1 \mathrm{mM}$ phenylmethylsulfonyl fluoride). The protoplasts were washed twice in a solution of $2 \mathrm{mM}$ MES, pH $5.7,154 \mathrm{mM} \mathrm{NaCl}, 125 \mathrm{mM}$ $\mathrm{CaCl}_{2}$ and $5 \mathrm{mM} \mathrm{KCl}$. For immunostaining, the protoplasts were treated with $0.05 \mu \mathrm{mol} \mathrm{NF}$ and $22 \mu \mathrm{g} \mathrm{ml}^{-1} \mathrm{Lin}$ for $90 \mathrm{~min}$ in the presence or absence of CHX followed by fixation with $2 \%(\mathrm{w} / \mathrm{v})$ paraformaldehyde for $15 \mathrm{~min}$. Subsequently, the protoplasts were blocked with PBS $\left(137 \mathrm{mM} \mathrm{NaCl}, 2.7 \mathrm{mM} \mathrm{KCl}, 10 \mathrm{mM} \mathrm{Na} \mathrm{HPO}_{4}\right.$ and $1.76 \mathrm{mM} \mathrm{KH}_{2} \mathrm{PO}_{4}$ ) buffer containing $0.5 \%(\mathrm{w} / \mathrm{v}) \mathrm{BSA}$ and $0.001 \%(\mathrm{v} / \mathrm{v})$ Triton $\mathrm{X}-100$ for $0.5 \mathrm{~h}$ and incubated with the anti-N-PTM antibodies in PBS-containing $0.1 \%(\mathrm{w} / \mathrm{v}) \mathrm{BSA}$ for $4 \mathrm{~h}$ at $4^{\circ} \mathrm{C}$ with very gentle shaking. The protoplasts were then washed three times in PBS-containing 0.005\% (v/v) Triton X-100 (PBST) followed by a $2 \mathrm{~h}$ incubation with FITC-conjugated anti-rabbit secondary antibody (Molecular Probes; $1: 200)$ in PBS-containing $0.5 \%(\mathrm{w} / \mathrm{v}) \mathrm{BSA}$ at $4^{\circ} \mathrm{C}$ with very gentle shaking. After three washes with PBST, the protoplasts were mounted on a glass slide in $50 \%$ (w/v) glycerol in Tris, $\mathrm{pH} 9.5$, containing an antifade reagent (Bio-Rad) and $0.1 \mu \mathrm{g} \mathrm{ml}^{-1} 4,6$-diamidino-2-phenylindole ${ }^{47}$. Immunofluorescence was examined using a confocal laser scanning microscope (LSM510; Carl Zeiss).

PTM processing. For the HL treatment, 4-week-old detached Arabidopsis leaves, floating adaxial side up on water, were illuminated with a photon flux density of $1,600 \mu \mathrm{mol} \mathrm{m}^{-2} \mathrm{~s}^{-1}$ for various number of hours in the presence or absence of CHX. For treatments with NF and Lin, the Arabidopsis seedlings were grown in the $1 / 2 \mathrm{LS}$ media in the presence of $220 \mu \mathrm{g} \mathrm{ml}^{-1} \mathrm{Lin}$ and $5 \mu \mathrm{M} \mathrm{NF}$. For treatments with protease inhibitors, pefabloc SC (4-(2-aminoethyl)-benzensulfonyl-flouride hydrochloride; $1 \mathrm{mg} \mathrm{ml}^{-1}$, Roche) or pepstatin A (1 $\mathrm{g} \mathrm{ml}^{-1}$, Sigma-Aldrich) was added under normal light condition for $1 \mathrm{~h}$ before HL treatments.

\section{References}

1. Leister, D. Chloroplast research in the genomic age. Trends Genet. 19, 47-56 (2003).

2. Soll, J. \& Schleiff, E. Protein import into chloroplasts. Nat. Rev. Mol. Cell Biol. 5, 198-208 (2004)

3. Cline, K. \& Dabney-Smith, C. Plastid protein import and sorting: different paths to the same compartments. Curr. Opin. Plant Biol. 11, 585-592 (2008).

4. Kessler, F. \& Schnell, D. Chloroplast biogenesis: diversity and regulation of the protein import apparatus. Curr. Opin. Cell Biol. 21, 494-500 (2009).

5. Li, H. M. \& Chiu, C. C. Protein transport into chloroplasts. Annu. Rev. Plant Biol. 61, 157-180 (2010).

6. Wollman, F. A., Minai, L. \& Nechushtai, R. The biogenesis and assembly of photosynthetic proteins in thylakoid membranes. Biochim. Biophys. Acta 1411, 21-85 (1999)

7. Gray, J. C., Sullivan, J. A., Wang, J. H., Jerome, C. A. \& MacLean, D. Coordination of plastid and nuclear gene expression. Philos. Trans. R. Soc. Lon. B Biol. Sci. 358, 135-144 (2003). 
8. Nott, A., Jung, H. S., Koussevitzky, S. \& Chory, J. Plastid-to-nucleus retrograde signaling. Annu. Rev. Plant Biol. 57, 739-759 (2006).

9. Pesaresi, P., Schneider, A., Kleine, T. \& Leister, D. Interorganellar communication. Curr. Opin. Plant Biol. 10, 600-606 (2007).

10. Kleine, T., Voigt, C. \& Leister, D. Plastid signaling to the nucleus: messengers still lost in the mists? Trends Genet. 25, 185-192 (2009).

11. Pogson, B. J., Woo, N. S., Förster, B. \& Small, I. D. Plastid signaling to the nucleus and beyond. Trends Plant Sci. 13, 602-609 (2008).

12. Woodson, J. D. \& Chory, J. Coordination of gene expression between organellar and nuclear genomes. Nat. Rev. Genet. 9, 383-395 (2008).

13. Bradbeer, J. W., Atkinson, Y. A., Börner, T. \& Hagemann, R. Cytoplasmic synthesis of plastid polypeptides may be controlled by plastid-synthesized RNA. Nature 279, 816-817 (1979).

14. Johanningmeier, U. \& Howell, S. H. Regulation of light-harvesting chlorophyllbinding protein messenger-rna accumulation in ChlamydomonasReinhardipossible involvement of chlorophyll synthesis precursors. J. Biol. Chem. 259, 13541-13549 (1984).

15. Strand, A., Asami, T., Alonso, J., Ecker, J. R. \& Chory, J. Chloroplast to nucleus communication triggered by accumulation of Mg-protoporphyrin IX. Nature 421, 79-83 (2003).

16. Oelmüller, R., Levitan, I., Bergfeld, R., Rajasekhar, V. K. \& Mohr, H. Expression of nuclear genes as affected by treatments acting on the plastids. Planta 168, 482-492 (1986).

17. Susek, R. E., Ausubel, F. M. \& Chory, J. Signal transduction mutants of Arabidopsis uncouple nuclear $C A B$ and $R B C S$ gene expression from chloroplast development. Cell 74, 787-799 (1993).

18. Mochizuki, N., Brusslan, J. A., Larkin, R., Nagatani, A. \& Chory, J. Arabidopsis genomes uncoupled 5 (GUN5) mutant reveals the involvement of Mg-chelatase $\mathrm{H}$ subunit in plastid-to-nucleus signal transduction. Proc. Natl Acad. Sci. USA 98, 2053-2058 (2001)

19. Larkin, R. M., Alonso, J. M., Ecker, J. R. \& Chory, J. GUN, a regulator of chlorophyll synthesis and intracellular signaling. Science 299, 902-906 (2003).

20. Shang, Y. et al. The Mg-chelatase $\mathrm{H}$ subunit of Arabidopsis antagonizes a group of WRKY transcription repressors to relieve ABA-responsive genes of inhibition. Plant Cell 22, 1909-1935 (2010).

21. Shen, Y. Y. et al. The Mg-chelatase $\mathrm{H}$ subunit is an abscisic acid receptor. Nature 443, 823-826 (2006).

22. Moulin, M., McCormac, A. C., Terry, M. J. \& Smith, A. G. Tetrapyrrole profiling in Arabidopsis seedlings reveals that retrograde plastid nuclear signaling is not due to Mg-protoporphyrin IX accumulation. Proc. Natl Acad. Sci. USA 105, 15178-15183 (2008).

23. Mochizuki, N., Tanaka, R., Tanaka, A., Masuda, T. \& Nagatani, A. The steadystate level of Mg-protoporphyrin IX is not a determinant of plastid-to-nucleus signaling in Arabidopsis. Proc. Natl Acad. Sci. USA 105, 15184-15189 (2008).

24. Escoubas, J., Lomas, M., LaRoche, J. \& Falkowski, P. G. Light-intensity regulation of $c a b$ gene transcription is signaled by the redox state of the plastoquinone pool. Proc. Natl Acad. Sci. USA 92, 10237-10241 (1995).

25. Apel, K. \& Hirt, H. Reactive oxygen species: metabolism, oxidative stress, and signal transduction. Annu. Rev. Plant Biol. 55, 373-399 (2004).

26. Fey, V. et al. Retrograde plastid redox signals in the expression of nuclear genes for chloroplast proteins of Arabidopsis thaliana. J. Biol. Chem. 280, 5318-5328 (2005).

27. Bräutigam, K. et al. Dynamic plastid redox signals integrate gene expression and metabolism to induce distinct metabolic states in photosynthetic acclimation in Arabidopsis. Plant Cell 21, 2715-2732 (2009).

28. Koussevitzky, S. et al. Signals from chloroplasts converge to regulate nuclear gene expression. Science 316, 715-719 (2007).

29. Hoppe, T., Rape, M. \& Jentsch, S. Membrane-bound transcription factors: regulated release by RIP or RUP. Curr. Opin. Cell Biol. 13, 344-348 (2001).

30. Chen, Y. N., Slabaugh, E. \& Brandizzi, F. Membrane-tethered transcription factors in Arabidopsis thaliana: novel regulators in stress response and development. Curr. Opin. Plant Biol. 11, 695-701 (2008).

31. Seo, P. J., Kim, S. G. \& Park, C. M. Membrane-bound transcription factors in plants. Trends Plant Sci. 13, 550-556 (2008).

32. Doerks, T., Copley, R. \& Bork, P. DDT-a novel domain in different transcription and chromosome remodeling factors. Trends Biochem. Sci. 26, 145-146 (2001).
33. Capili, A. D., Schultz, D. C., Rauscher, I. F. \& Borden, K. L. Solution structure of the PHD domain from the KAP-1 corepressor: structural determinants for PHD, RING and LIM zinc-binding domains. EMBO J. 20, 165-177 (2001).

34. Horton, P. et al. WoLF PSORT: protein localization predictor. Nucleic Acids Res. 35, W585-W587 (2007).

35. de la Paz Sanchez, M. \& Gutierrez, C. Arabidopsis ORC1 is a PHD-containing H3K4me3 effector that regulates transcription. Proc. Natl Acad. Sci. USA 106, 2065-2670 (2009)

36. Peña, P. V. et al. Molecular mechanism of histone H3K4me3 recognition by plant homeodomain of ING2. Nature 442, 100-103 (2006).

37. Shi, X. B. et al. ING2 PHD domain links histone H3 lysine 4 methylation to active gene repression. Nature 442, 96-99 (2006).

38. Wysocka, J. et al. A PHD finger of NURF couples histone H3 lysine 4 trimethylation with chromatin remodelling. Nature 442, 86-90 (2006).

39. Sims, R. J. \& Reinberg, D. Histone H3 Lys 4 methylation: caught in a bind? Genes Dev. 20, 2779-2786 (2006).

40. Zimmermann, P., Hirsch-Hoffmann, M., Hennig, L. \& Gruissem, W. GENEVESTIGATOR. Arabidopsis microarray database and analysis toolbox. Plant Physiol. 136, 2621-2632 (2004).

41. Woodson, J. D., Perez-Ruiz, J. M. \& Chory, J. Heme synthesis by plastid ferrochelatase I regulates nuclear gene expression in plants. Curr. Biol. 21, 897-903 (2011)

42. Lin, R. C., Ding, L., Casola, C., Ripoll, D. R., Feschotte, C. \& Wang, H. Y. Transposase-derived transcription factors regulate light signaling in Arabidopsis. Science 318, 1302-1305 (2007)

43. Yoo, S. D., Cho, Y. H. \& Sheen, J. Arabidopsis mesophyll protoplasts: a versatile cell system for transient gene expression analysis. Nat. Protoc. 2, 1565-1572 (2007)

44. Leibfried, A. et al. WUSCHEL controls meristem function by direct regulation of cytokinin-inducible response regulators. Nature 438, 1172-1175 (2005)

45. Ouyang, M. et al. LTD is a protein required for sorting light-harvesting chlorophyll-binding proteins to the chloroplast SRP pathway. Nat. Commun. 2:277 doi: 10.1038/ncomms1278 (2011).

46. Keegstra, K. \& Youssif, A. E. Isolation and characterization of chloroplast envelope membranes. Methods Enzymol. 118, 316-325 (1986)

47. Chuong, S. D., Franceschi, V. R. \& Edwards, G. E. The cytoskeleton maintains organelle partitioning required for single-cell C4 photosynthesis in Chenopodiaceae species. Plant Cell 18, 2207-2223 (2006).

\section{Acknowledgements}

We are grateful to ABRC for the Arabidopsis seeds. This research was supported by the National Natural Science Foundation of China (30725003), the Major State Basic Research Development Program (2009CB118500), the Frontier Project of the Knowledge Innovation Engineering (KSCX2-EW-J-1) and Solar Energy Initiative of the Chinese Academy of Sciences. We thank Professor Weicai Yang for help in immunofluorescence analysis and Professor James Barbers for helpful comments.

\section{Author contributions}

X.S. and L.Z. designed the study; X.S., P.F., X.X., H.G., J.M. and W.C. performed the research; X.S., R.L., C.L. and L.Z. analysed the data; X.S. and L.Z. wrote the paper. All authors discussed the results and made comments on the manuscript.

\section{Additional information}

Supplementary Information accompanies this paper at http://www.nature.com/ naturecommunications

Competing financial interests: The authors declare no competing financial interests.

Reprints and permission information is available online at http://npg.nature.com/ reprintsandpermissions/

How to cite this article: Sun, X. et al. A chloroplast envelope-bound PHD transcription factor mediates chloroplast signals to the nucleus. Nat. Commun. 2:477 doi: 10.1038/ncomms1486 (2011) 